\title{
Structural analysis of molecular cloud maps: the case of the star forming Vela-D cloud
}

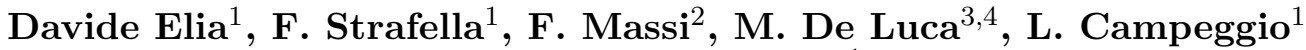 \\ and B. M. T. Maiolo ${ }^{1}$ \\ ${ }^{1}$ Dipartimento di Fisica, Università di Lecce, CP 193, I-73100 Lecce, Italy \\ email: eliad@le.infn.it \\ ${ }^{2}$ INAF - Osservatorio Astrofisico di Arcetri, Largo E. Fermi 5, 50125 Firenze, Italy \\ ${ }^{4}$ Dipartimento di Fisica, Università degli Studi di Roma "Tor Vergata", Via della Ricerca \\ Scientifica 1, I-00133 Roma, Italy \\ ${ }^{3}$ INAF - Osservatorio Astronomico di Roma, via Frascati 33, 00040 Monteporzio Catone, Italy
}

\begin{abstract}
We present the preliminary results of a statistical analysis carried out on a $1^{\circ} \times$ $1^{\circ} \mathrm{CO}(1-0)$ map of the intermediate mass star forming region Vela-D Cloud. Our goal is to determine statistical parameters suitable to quantify the structure of the observed cloud, in particular the power-law exponent of the map power spectrum. Furthermore, to help in removing the degeneracy implied in using a single parameter, we also resort to the multifractal approach.
\end{abstract}

Keywords. turbulence, ISM: clouds, structure, stars: formation

In last decades, several statistical tools were developed in order to characterize the structure of the molecular cloud maps obtained in the radio, submm and IR domains, and to infer on the 3D mass distribution. The comparison of the results obtained for maps which can differ in observing technique, wavelength, size, resolution, physical conditions, galactic position, fluidodynamical regime and presence of star formation can offer a way to better understand the differences observed in the star forming activity. Here we discuss the case of the SEST CO(1-0) observations of Vela-D Cloud presented and widely described in Elia, Massi, Strafella, et al. 2006. We applied the robust method of the $\Delta$-variance (Stutzki, Bensch, Heithausen, et al. 1998) to determine the power-law exponent $\beta$ of the power spectrum of the integrated intensity map from -2 to $20 \mathrm{~km} \mathrm{~s}^{-1}$ and of the single channel maps obtained integrating over intervals of $3 \mathrm{~km} \mathrm{~s}^{-1}$ starting from $2 \mathrm{~km} \mathrm{~s}^{-1}$. We obtained $\beta=2.78$, corresponding to a fractal dimension $D=2.60$. As indicated by Stutzki, Bensch, Heithausen, et al. (1998), this parameter can be related with the clump mass spectrum of the investigated cloud (Elia, Massi, Strafella, et al. 2006), suggesting a scenario in which clumps have, on average, constant column density. Moreover, we found $\beta=2.51,2.87,2.89,2.77$ for the four channels map, respectively. As a further preliminary result, we found that the multifractal spectrum calculated as in Chappell \& Scalo (2001) (but considering here also negative orders) for the above mentioned maps of Vela-D Cloud appears to be wider for the integrated intensity map than for the single channel maps, in particular for negative orders.

\section{References}

Chappell, D. \& Scalo, J.M. 2001, ApJ 551, 712

Elia, D., Massi, F., Strafella, F., De Luca, M., Giannini, T., Lorenzetti, D., Nisini, B., Campeggio, L., \& Maiolo, B.M.T. 2006, ApJ submitted

Stutzki, J., Bensch, A., Heithausen, A., Ossenkopf, V., \& Zielinsky, M. 1998, A\& A 336, 697 